\title{
Renewable energy allocation based on maximum flow modelling within a microgrid
}

\author{
Junghoon Lee, Gyung-Leen Park \\ Department of Computer Science and Statistics, Jeju National University, Jeju City, Republic of Korea
}

\begin{tabular}{|c|c|}
\hline Article Info & ABSTRACT \\
\hline Article history: & \multirow{9}{*}{$\begin{array}{l}\text { This paper designs a microgrid-wide energy allocation mechanism on top of } \\
\text { a network flow model from distributed generators to consumer entities. } \\
\text { Basically, the flow graph consists of a set of nodes representing consumers } \\
\text { or generators as well as a set of weighted links representing the amount of } \\
\text { energy generation, consumer-side demand, and transmission cable capacity. } \\
\text { The main idea lies in that a special node is added to account for the } \\
\text { interaction with the main grid and that two-pass allocation is executed. In the } \\
\text { first pass, the maximum flow solver decides the amount of the insufficiency, } \\
\text { which must be supplemented by the main power network, usually with } \\
\text { predefined cost. The second pass runs the flow solver again to fill the energy } \\
\text { lack and calculates the surplus of renewable energy generation. The } \\
\text { experiment result observes the stability in energy distribution over the } \\
\text { microgrid while the amount of the total energy production can be } \\
\text { accommodated by the maximum link capacity. }\end{array}$} \\
\hline Received Apr 30, 2021 & \\
\hline Revised Jul 17, 2021 & \\
\hline Accepted Aug 15, 2021 & \\
\hline Keywords: & \\
\hline 2-pass allocation & \\
\hline Microgrid & \\
\hline Network flow model & \\
\hline Renewable energy & \\
\hline
\end{tabular}

This is an open access article under the CC BY-SA license.

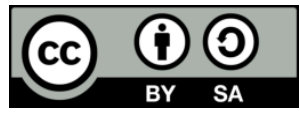

\section{Corresponding Author:}

Gyung-Leen Park

Department of Computer Science and Statistics, Jeju National University

Jejudaehakno 102, Republic of Korea

Email: glpark@jejunu.ac.kr

\section{INTRODUCTION}

Smart cities are developing a variety of leading-edge services to be provided to citizens, empowered by the fully matured information technologies such as big data analysis and artificial intelligence techniques in addition to the classic computer algorithms [1]. As one of the most important services in those smart cities, smart grids are leveraging the legacy power network on top of the ever-growing computational intelligence to achieve energy efficiency, greenhouse gas reduction, and many others [2]. Most of all, the data communication infrastructure, including both wireless and wired channels, is the most fundamental to the smart grid, as it allows a variety of energy entities to exchange messages correctly in real time, decide an appropriate control action and perform the issued command cooperatively for the given system goal [3]. Energy entities play their roles on the power network from generation and distribution to consumption. So, a lot of entities comprise the whole power system and interact in an extremely complex manner. Moreover, each of them creates a massive volume of monitoring data, making essential an elaborate data analysis and planning mechanism.

In modern power systems, new entities keep appearing. First, renewable energy such as solar and wind power, is extending its coverage in electricity markets. While such energy can reduce air pollution mainly coming from carbon dioxide $\left(\mathrm{CO}_{2}\right)$ emissions, its energy source is not consistently available and it is quite difficult to efficiently allocate energy to consumers. Second, electric vehicles (EVs) are gradually penetrating into our daily lives, replacing gasoline powered vehicles [4]. They are inherently embedding battery devices and can be charged even with the intermittency of the energy source [5]. Third, the energy 
storage capability supported by battery devices makes it possible to shift peak load and allows diverse applications such as bidirectional energy trade [6]. However, EVs impose a complex and unprecedented load on the grid, and their simultaneous charging will sharply increase the energy demand. Yet, this load is somewhat controllable, while it may sacrifice the user-side comfort, for example, the extension of charging time due to squeezed electricity injection.

In addition, as subsystems of the main grid, microgrids operate quite autonomously, generating, and consuming energies in their own ways. From the viewpoint of the main grid, microgrids are a new type of unpredictable load. Moreover, a microgrid may contain renewable energy generators and EV chargers in addition to the legacy power elements [7]. It tries to control energy generation and consumption behaviors according to consumer requirements, energy source availability, and the interaction strategy with the main grid. The unavoidable intermittency of renewable energy should be overcome so as to enhance the energyindependency of a target microgrid [8]. It is clear that the massive penetration of EVs can significantly reduce the creation of greenhouse gas, as the transport is the most responsible source of $\mathrm{CO}_{2}$ emissions [9]. Hence, microgrids are required to try to accommodate as many EVs as possible, making their charging more convenient and energy-efficient. Those efforts may include charging facility siting, energy provisioning, and driver-friendly charger reservation services [10], [11]. In addition, scheduled energy consumption, combined with the real-time price adaptation, can reduce the peak load, and avoid the construction of new power plants.

Even though a microgrid wants to work autonomously as much as possible, it may suffer from not only energy shortage but also temporal overproduction [12]. Hence, uncontrolled energy flow to and from the main grid may jeopardize the system safety. As the tight control of the energy flow between both grids is quite complicated and expensive, it is necessary to maximize the consumption of generated renewable energy within the microgrid, suppressing the energy flow from the main power network. Renewable energy can flow to a consumer entity only when there exists a direct cable connection from a generator to itself. Practically not every generator and consumer entity are connected to the main energy system due to some reasons such as geographic constraints, lack of frequency adaptation, or economic reasons [13]. As a result, some consumer devices run with the energy just from a subset of generators, experiencing the pause and resumption of their operations. After all, the energy consumption behavior in a microgrid subsystem can be viewed as a network flow graph, and thus we can develop an efficient energy matching mechanism for the specific target microgrid. An efficient energy matching enhances the independency of the microgrid by maximizing the consumption amount of self-creating renewable energy.

In the meantime, Figure 1 plots the distribution of EV charging stations and solar energy generators in Jeju city, Republic of Korea. The perimeter of this island is about $200 \mathrm{~km}$. In the Figure 1, circles denote the locations of charging stations while crosses those of solar generation facilities. Not every element, namely, generator or charging station, is connected to the main grid and the energy generated by solar panels must be consumed by nearby EV charging stations or facilities. Moreover, some renewable energy generators cannot send electricity to the main grid. In this situation, a set of geographically close stations and generators can form a microgrid which has its own control and management policy regarding the installation of new transmission cables, energy provisioning, renewable energy allocation, forecasting of energy generation and charging demand, coordination with the main grid, and the like [14]. In this regard, this paper is to design an energy allocation scheme for such a power network to enhance the autonomous control within a microgrid and minimize the additional energy purchase from the main grid. As an extended version of our previous paper [15], this paper generalizes the power consumption scenario and includes the extensive performance measurement result.

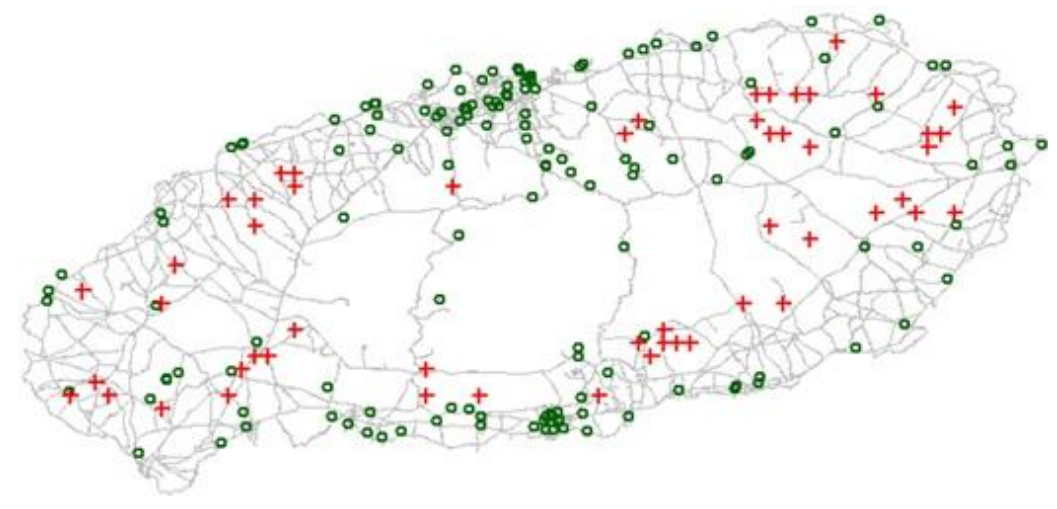

Figure 1. Distribution of charging stations and solar power generators 
This paper is organized as follows: after issuing the problem in section 1, section 2 reviews some related work. Then, section 3 describes important assumptions and designs an energy allocation scheme consisting of 2 passes on top of network flow modeling. After the performance of the proposed scheme is measured in section 4 , section 5 concludes this paper with a brief introduction of future work.

\section{RESEARCH METHOD}

\subsection{Background and related work}

The intermittency of renewable energy is the main hurdle to its wide deployment, but this problem can be significantly overcome when consumption entities accommodate a demand response mechanism. For example, a smart building is generally equipped with an energy storage system and thus absorb the peak load by discharging the stored energy instead of getting energy from the main grid. In addition, EVs in a charging station are inherently battery devices and they can adjust their time-of-charging. Hence, we can estimate the energy demand during the specific time interval, which necessarily coincides with the length of the real-time price change, and then provision the needed energy. For example, Al Hadi et al. [16] suggests an efficient demand response algorithm capable of controlling the load based on the current power availability, electricity price, consumer-side comfort level, and appliance priority. It exploits a heuristic-based evolutionary algorithm with the optimization goal set to load shifting. The demand response scheme works fully dynamically according to the context defined by predicted generation, load profile, and current state-ofcharge of the electricity battery. This approach is mainly targeting at a smart building which needs energy for lighting, heating, ventilating, and air conditioning.

Demand side management (DSM) allows a demand response mechanism to work more efficiently and automatically. It supports electricity market control, distributed energy resource coordination, and most importantly, direct energy demand adjustment. Electricity load is reshaped on the target grid by peak clipping, valley filling, load shifting, and other techniques [17]. Among these, the load shifting method is most widely exploited, moving the controllable load such as EV charging from peak time to off-peak time. Other methods tend to just cut down the overall energy consumption when necessary. A heuristic-based load shift scheme is also proposed to cope with the ever-growing number of consumer devices and renewable energy sources. It is assumed that the management entity decides the consumption schedule usually a day advance, while the control action is issued and delivered on real-time basis. The management process takes desired load curve as input and decides the consumption plan to be as similar to it as possible.

As an example of inter-grid cooperation, Xia et al. [18] proposes a hierarchical control mechanism performing primary and secondary control across a microgrid and a weak grid. Here, a weak grid usually suffers from instability in voltage or frequency, which mainly comes from absence of control capability. Especially, unpredictable fluctuations in wind power generation bring severe instability to the grid. The authors design a system consisting of local and central controllers while the battery unit plays a core role. In the main grid, a dispatch center orders the predefined secondary frequency regulation to the battery unit to supply the stored energy on the under-frequency condition. On the contrary, in the case of over-frequency, the primary frequency control makes the battery unit absorb the surplus, invoking a pitch calibration in the secondary control. Their research also includes how to estimate the minimum battery capacity to meet the given requirement and the minimum battery charging power.

Most microgrids operate in both grid-connected and isolated modes [19]. When connected to the main grid, mainly via the point of common coupling, a microgrid turns into a subsystem containing autonomous electricity load, renewable energy generation, and electricity storage. Aviles et al. [19] designs a fuzzy logic controller embedding just 25 rules to manage non-controllable renewable energy production and electricity load. The main objective lies in smoothing the energy exchange between the two grids to reduce the peak load, to minimize the demand fluctuation and to keep the battery charger level in a secure state. The logic makes the high-frequency component be exchanged with the battery while the low-frequency component with the main grid. Like this, the main grid can compensate for the insufficiency of available energy in the microgrid but the amount must be made as small as possible.

EV charging control, fully cooperating with other grid entities, is required to perform the local energy optimization along with the renewable energy generation. When connected to the main grid, it is necessary to check the load imbalance, harmonic disturbances, and the potential of possible renewable energy sources for the decision of hosting capacity. Lamedica et al. [9] overviews the methods regarding hosting capacity estimation and EV charging control schemes. Existing control schemes usually take as input status of charge (SoC) level, desired end time, charging cost, network congestion, local voltage, and distributed generation, be it local or global coordination. The goals of the charging control are voltage regulation and load shifting in any unpredictable situations. The authors propose a scheduling-based control scheme which decides the charging operation to maintain the total harmonic distortion at the point of common coupling 
within the permissible bound. To this end, the control system keeps monitoring the number of EVs that of EVs tripped from overload condition and their SoCs.

\subsection{Network flow scenario}

The energy flow from generators to consumers in a microgrid is abstracted by a uni-directed graph from a virtual source node to a virtual sink node, while generators and consumers are taken as general nodes as depicted in Figure 2. Here, it is sufficient to show only those consumer nodes associated with at least one of renewable sources. If a consumer takes electricity solely from the main grid, it is not our concern. Source and sink are virtual nodes which do not denote actual player of the energy network but they abstract the allocation process. The virtual source has outbound links to all respective generator nodes while the virtual sink node has inbound links from all respective consumers. In addition to the source and sink, the graph model embraces two bipartite constituents, which can be found in two separate columns. The first group represents the generators and the other is the consumers. Links connects generators to consumers, according to whether a transmission cable physically established both entities.

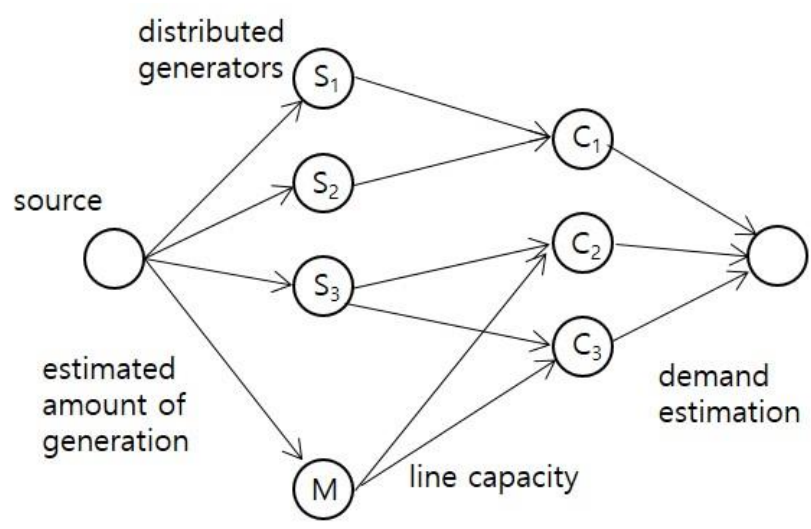

Figure 2. Network flow model

In the flow graph, each link has its own weight, which is decided by the actual energy transfer in a microgrid. First, the link weight from the source to a generator node, such as $S_{1}, S_{2}$, and $S_{3}$ in Figure 2, is the amount of electricity produced in the generator, possibly during the specific time interval. This value is constrained by its maximum generation capacity, which depends on the performance of solar panels, battery chargers, and the like. The commonly available solar energy facility in these days can generate tens or hundreds of kilowatt $(\mathrm{kW})$. Our scheme employs a special node, $\mathrm{M}$, to represent the energy flow from the main grid. It belongs to the generator group and has a link from the source node with the capacity decided by the amount of contracted and provisioned energy. M receives electricity through the main grid generally from stable sources such as thermal or nuclear power plants. Locally generated electricity can be distributed to a set of consumers. According to the cable layout, a single consumer can take from multiple and possibly heterogeneous generators. In addition, the link weight from a power generator to a consumption entity is necessarily limited by the transmission capacity of the cable installation.

In the meantime, the energy demand of a consumer entity, such as $\mathrm{C}_{1}, \mathrm{C}_{2}$, or $\mathrm{C}_{3}$ in Figure 2 , is denoted by the weight of its link to the sink node [20]. For a consumer entity, the sum of all input link weights corresponds to the total energy supplied by all connected sources and it must be larger than the weight of its outgoing link to be able to satisfy the energy demand. Here, the whole electricity system is stable when this condition is met for all nodes in the microgrid except those taking energy just from a renewable energy generator such as $\mathrm{C}_{1}$. The power system may fail to satisfy the energy demand when the renewable energy generation is not sufficient. Here, the inbound flow is less than the actual demand irrespective of the installed electricity transmission potential on the inappropriate energy generation condition such as bad weather. In that case, $\mathrm{C}_{1}$, which may be the battery-equipped facility, will highly likely start discharging its battery, work in the energy-saving mode, or suspend its operation. As contrast, $\mathrm{C}_{2}$ and $\mathrm{C}_{3}$, taking energy from both the main grid and local sources, just tries to more consume the energy created from the latter.

While the link weight is limited by the grid infrastructure such as cable capacity and generation capability, the actual energy flow changes according to the dynamic features in the energy demand and availability. To begin with, the amount of solar energy generation, which is represented by the link weight 
from the source to a generator, changes according to the climate condition such as current radiation strength and cloudiness [21]. For a generator node, the sum of output flows to consumers denotes the amount of energy produced at the generator and then allocated to relevant consumers. The allocation should match the consumer-side demand. In the allocation, the sum of flows to a specific consumer node cannot exceed the current consumer-side demand, which is the weight of link from a consumer to the sink. This amount also changes according to the real-time consumption of energy depending on the operation status of the devices.

This model allows us to build a microgrid-level provisioning plan after the link-by-link flow calculation supported by generally available computer power [22]. Contrary to the maximum link weight fixed by the hardware component, actual flows are updated on each time interval. The flow weight from the virtual source to a generator node can be predicted, exploiting a weather forecast scheme currently available in many areas. Generally speaking, the renewable energy generation much depends on the daily climate condition change. In addition, the energy demand is also forecasted for each consumer object based on its consumption levels during the previous periods and reserved operation schedule. On the other hand, power demand keeps changing dynamically in microgrid level as the consumption of a lot of devices are integrated. However, the energy storage equipment can possibly absorb such demand fluctuations, making the load sufficiently stable, or at least piecewise constant. Hence, the allocation procedure desirably takes the average demand level.

Without the existence of node $\mathrm{M}$, the allocation of renewable energy generation can be allocated by the classic maximum flow algorithm. However, we are trying to minimize the energy purchase from the microgrid not just mathematically distribute the energy. The link weight from the source to $M$ can be as much as the maximum amount of energy flow contracted between the main grid and the microgrid. The link weight from $\mathrm{M}$ to most consumption nodes must be large enough to meet their consumer demands even in the absence of renewable energy for their ceaseless operations. Figure 3 shows an example of the operation of the maximum flow solver. In the Figure 3(a), $\mathrm{S}_{1}$ generates 5 units of energy (upper bound is not shown), while it can send to $C_{1}$ up to 5 and to $C_{2}$ up to 4 , while $C_{1}$ needs 4 units. After the execution of the maximum flow solver, 2 units independently go to $C_{1}$ and $C_{2}$, as shown in Figure 3(b). 1 unit, stuck at $S_{1}$ can be thrown away or send back to and offset the complement from the main grid. For each link in the graph, the flow allocation denoted in Figure 3(b) must be less than the link capacity fixed in Figure 3(a).

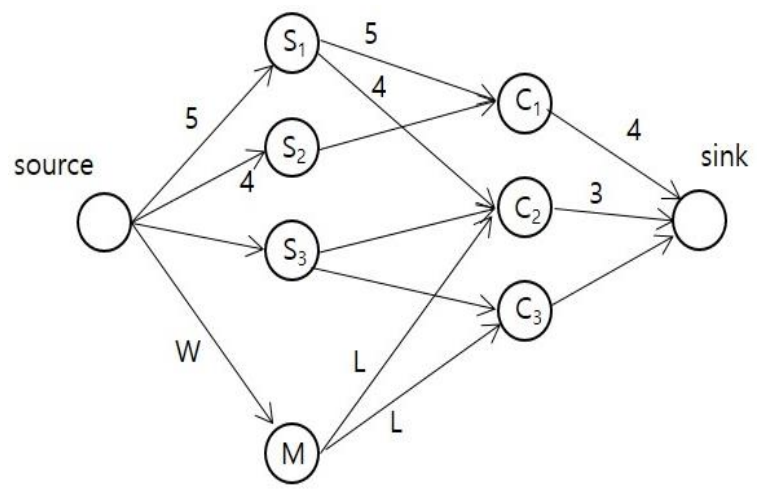

(a)

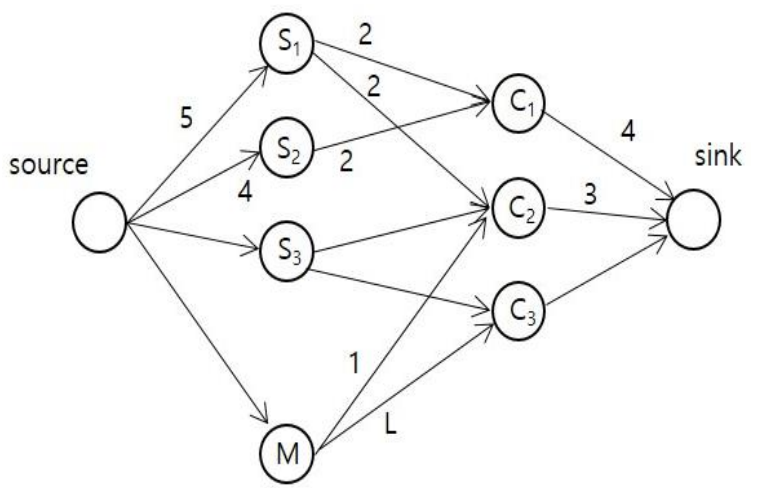

(b)

Figure 3. Flow modeling on (a) link capacity and (b) the amount of actual flow 


\subsection{2-pass allocation}

Formally, a flow network means a network of nodes and links, additionally having a source and a sink. Except source and sink nodes, every node can receive and send an equal amount of stuff through it. The source only sends and the sink only receives the flow. A flow network is represented by a weighted directional graph and the weight of each link decides the maximum amount of flow between the connected two nodes. Finding the network-wide maximum flow from the source to the sink allows us to efficiently allocate the available resource to the appropriate place. It must be mentioned that a weight may have a nonfixed value. Then, the weight is set to its upper bound, making unavoidable over-allocation and energy waste. We can convert the graph which abstracts generators and consumers in the physical world, to the appropriate data structure to implement computer algorithms in the cyber world.

After the weight of each link is determined, we can estimate the maximum amount of locally consumable renewable energy, with the Ford-Fulkerson scheme [23]. It adds a reverse line for each link for the possible subsequent reclamation of the weight coming from path canceling, even though Figure 3 does not show those reverse links. This scheme repeats finding a path from source to sink, until there is no further path. In each iteration of finding a new path, the amount of feasible energy flow for a path is estimated by the minimum of the weights along the path. The link weights along the path are decreased by this minimum value. Here, the process also increases the weight of every reverse link, which can be the feasible path from the source to the sink in the subsequent path finding step. When a reverse link is included in the path, the previous weight allocation is canceled.

At each allocation step, the link weights from the source to generators $\left(\mathrm{S}_{\mathrm{i}}\right.$ 's) and those from the consumers $\left(\mathrm{C}_{\mathrm{i}}\right.$ 's) to the sink are given based on the respective forecast mechanisms. The energy allocation scheme tries to decide the link weights from $S_{\mathrm{i}}$ 's to $\mathrm{C}_{\mathrm{i}}$ 's as well as the link weight from the source to $\mathrm{M}$, say $\mathrm{W}$. The first weight group focuses on how to distribute renewable energy generation to consumers, while W needs to be calculated to make up for the energy insufficiency, but this amount should be made as small as possible. To this end, we suggest a two-pass allocation scheme to meet this requirement.

The first pass sets $\mathrm{W}$ to a minimum flow contracted with the power system, say $\mathrm{W}_{\mathrm{L}}$, and runs the maximum flow solver. In most cases, up to $\mathrm{W}_{\mathrm{L}}$, the main grid provides the energy with the basic cheap rate and $\mathrm{W}_{\mathrm{L}}$ can be even zero. In the first pass, those consumers receiving energy just from renewable sources, such as $\mathrm{C}_{1}$ in Figure 3(a), must have precedence as their insufficiency cannot be compensated by the main grid. After the first pass, for each consumer node, we can calculate the amount of insufficiency by subtracting the sum of input link weights from its output link weight. If the energy demand is met just with renewable energy generation and basic grid-providing energy, the total sum of insufficiency terms will be zero. The microgrid can operate in isolated mode, disconnected from the main grid in case $\mathrm{W}_{\mathrm{L}}$ is zero. Otherwise, if for all consumers which have connection also with the main grid will be additionally purchased.

Next, for each consumer which takes energy also from the main grid, we can calculate the amount of insufficiency by subtracting the sum of the weights of its input links from its output link weight. Each insufficiency is added up to estimate the microgrid level lack of energy. It is necessary to additionally purchase from the main grid. Actually, even if this amount is supplied from the main grid, not every energy lack cannot be covered, due to cable connectivity or capacity. Moreover, not every energy from renewable sources can be used due to the same reason. However, to give the minimal possibility to meet the energy demand, the allocation procedure adds the total insufficiency to $\mathrm{W}$. Then, the maximum flow solver is executed again with modified $\mathrm{W}$, while other values remain the same as in the first pass. The link weights from $S_{i}$ 's to $C_{i}$ 's are the final allocation of energy to each consumer. The energy surplus taking place due to the inconsumable renewable energy generation can be send back to the main grid or just discarded.

\section{PERFORMANCE ANALYSIS RESULT ANALYSIS}

This section implements the proposed energy allocation model with the Java programming language to trace and estimate the actual energy flow from generators to consumers over the target microgrid. A virtual microgrid is set to have 10 solar energy generators and 20 consumer entities which are directly cableconnected from the solar energy sources, considering geographical clustering sizes. Without loss of generality, each consumer is assumed to demand the same units of energy, while the unit, for example, $\mathrm{kW}$, need not to be explicitly specified, as it has a different value according to the scale of enery productivity and consumption in the microgrid. In addition, the simulation employs an experiment parameter associated with cable provisioning and electricity pulling from the power system, $\mathrm{W}_{\mathrm{L}}$, and its default is set to 50 units. The maximum transmission capacity from a single generator to a single consumer is set to 10 units while each consumer needs 20 units. The experiment measures include the amount of electricity purchased from the main grid, amount of surplus generation, and the ratio of surplus energy. The performance parameters include amount of generated energy, average number of connections to the solar generators, and number of connections to the main grid. 
The first experiment observes the effect of the renewable energy generation, assuming that each generator creates the same amount of energy due to geographical affinity. The experiment changes pergenerator energy creation from 0 to 50 units, while each generator is connected to randomly select 2 consumers on average and only 3 consumers take electricity also from the main grid. When no energy is created from renewable source, only they can fully meet the energy demand. Until each generator creates 34 units, all the energy is sent to the consumers connected just to the distributed sources, as can be seen in Figure 4(a). In this case, the energy is produced beyond the demand and thus it is not necessary to purchase additionally. This result indicates the efficiency of the proposed network flow mechanism in allocating the intermittently produced energy to involved consumers, supported by the estimation of energy insufficiency, while its computation time lies within a reasonable range. Figure 4(b) and Figure 4(c) plot the dynamics of the surplus generation according to the per-generator energy creation. Here again, the surplus is almost zero until 16 units, as the energy generation is low and all energy is consumed within the microgrid. As the surplus can be predicted with the proposed model, it can be sold to other grid or stored in the battery device. The upper bound of the transmissible energy from a generator to a consumer prohibits the sufficient distribution.

Next experiment measures the effect of the average number of connections from a generator to consumers. In the experiment, per-generator energy creation is set to 30 units. Until the average number of connections reaches 4 , no energy can be transmitted to any consumers and the additional purchase remains at 60 units. A consumer entity can meet its demand only when all connected generators produce electricity, considering the rather restricted capacity of transmission cables between generators and consumers. So, the number of connections has little impact on the amount of electricity to purchase. In spite of the additional purchase, only 3 consumers can meet their demand. Essentially, as more consumers are connected, more energy can be delivered and used. However, there exists a saturation point, namely, 4 consumers, as shown in Figure 5(a). Beyond this point, every energy is consumed within the microgrid, no additional reduction is observed as can be seen in Figure 5(b) and Figure 5(c).

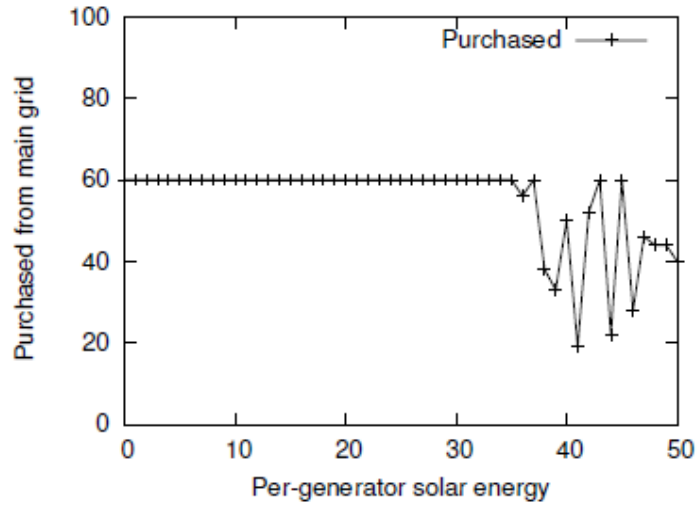

(a)

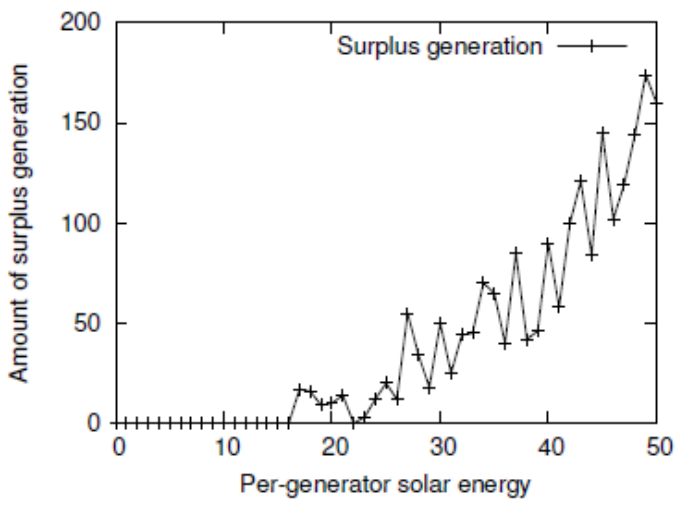

(b)

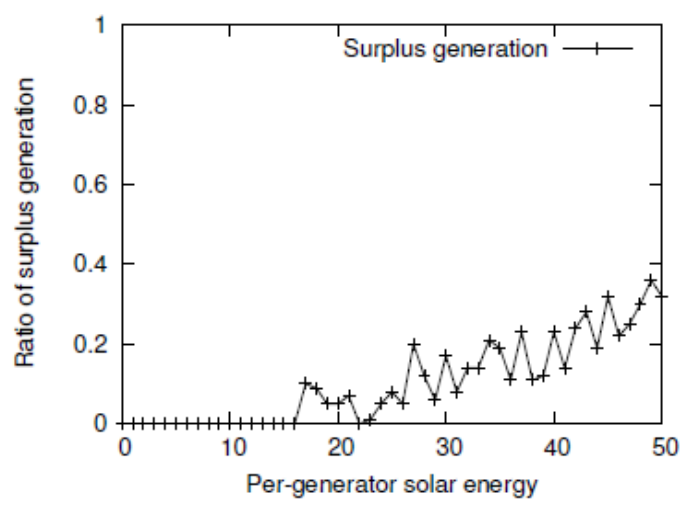

(c)

Figure 4. Effect of the amount of generated energy on (a) purchased electricity, (b) surplus generation, and (c) surplus ratio 


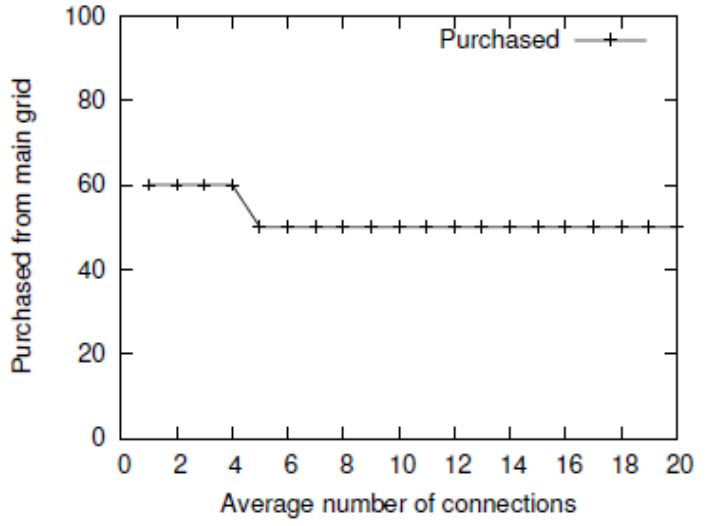

(a)

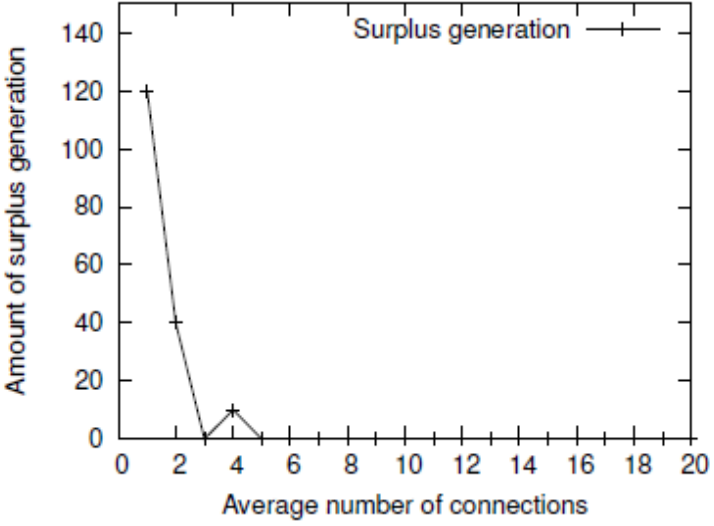

(b)

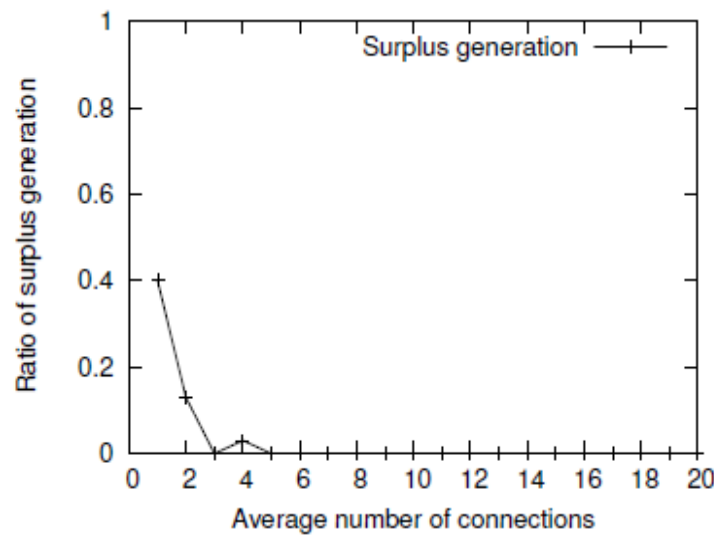

(c)

Figure 5. Effect of the average number of connections to solar generators on (a) purchased electricity, (b) surplus generation, and (c) surplus ratio

\section{CONCLUSION}

This paper has proposed a design and traced the performance of an energy allocation scheme for microgrids which keep exploiting more renewable energy generators and consumer facilities. The proposed model, beginning from network flow graph modeling, incorporates underlying electricity transmission capacity, grid-side supplement, and the energy behavior predictions regarding both energy gnerator and consumer demand sides. The proposed two-pass maximum flow mechanism first estimates how much electricity is needed from the main energy system to meet the overall demand considering the current level of local energy productivity. Here, the Ford-Fulkerson algorithm is exploited to estimate the amount of allocatable energy flow from generator nodes to a set of consumer nodes on the flow graph. The performance measurement results show that the proposed scheme can stably assign renewable energy to consumers and calculates the surplus, making it possible to establish an efficient energy plan for given parameter setting. As such, nowadays, sophisticated computer algorithms enrich smart services in the smart city. As a next stage of this paper, we are collecting the energy consumption profile from about $250 \mathrm{EV}$ (Electric Vehicle) chargers in our region and developing a demand forecast model for a geographical cluster of chargers. The renewable energy can be most efficiently consumed for EV charging as the intermittency of energy generation has almost no effect on charging battery devices.

\section{ACKNOWLEDGEMENTS}

This work was done while Prof. Gyung-Leen Park’s research year of Jeju National University in 2020.

\section{REFERENCES}

[1] J. J. Astrain, F. Falcone, A. Lopez, P. Sanchis, J. Villadangos, and I. R. Matias, "Monitoring of electric buses within an urban smart city environment," in Proceedings of IEEE Sensors, Oct. 2020, vol. 2020-Oct., doi: 
10.1109/SENSORS47125.2020.9278791

[2] J. M. Clairand, J. Rodriguez-Garcia, and C. Alvarez-Bel, "Assessment of technical and economic impacts of EV user behavior on EV aggregator smart charging,” Journal of Modern Power Systems and Clean Energy, vol. 8, no. 2, pp. 356-366, 2020, doi: 10.35833/MPCE.2018.000840.

[3] M. H. Cintuglu, O. A. Mohammed, K. Akkaya, and A. S. Uluagac, "A survey on smart grid cyber-physical system testbeds," IEEE Communications Surveys and Tutorials, vol. 19, no. 1, pp. 446-464, 2017, doi: 10.1109/COMST.2016.2627399.

[4] T. Morstyn, A. Teytelboym, and M. D. McCulloch, "Bilateral contract networks for peer-to-peer energy trading," IEEE Transactions on Smart Grid, vol. 10, no. 2, pp. 2026-2035, Mar. 2019, doi: 10.1109/TSG.2017.2786668.

[5] A. Azizivahed et al., "Energy management strategy in dynamic distribution network reconfiguration considering renewable energy resources and storage," IEEE Transactions on Sustainable Energy, vol. 11, no. 2, pp. 662-673, Apr. 2020, doi: 10.1109/TSTE.2019.2901429.

[6] H. Krueger and A. Cruden, "Multi-layer event-based vehicle-to-grid (V2G) scheduling with short term predictive capability within a modular aggregator control structure," IEEE Transactions on Vehicular Technology, vol. 69, no. 5, pp. 4727-4739, May 2020, doi: 10.1109/TVT.2020.2976035.

[7] Z. Cao, C. C. Chu, and R. Gadh, "An autonomous electric vehicle based charging system: Matching and charging strategy," in 2018 IEEE Power and Energy Society Innovative Smart Grid Technologies Conference, ISGT 2018, Feb. 2018, pp. 1-5, doi: 10.1109/ISGT.2018.8403365.

[8] Z. Shi, H. Liang, S. Huang, and V. Dinavahi, "Distributionally robust chance-constrained energy management for islanded microgrids," IEEE Transactions on Smart Grid, vol. 10, no. 2, pp. 2234-2244, Mar. 2019, doi: 10.1109/TSG.2018.2792322.

[9] R. Lamedica, A. Geri, F. M. Gatta, S. Sangiovanni, M. Maccioni, and A. Ruvio, "Integrating electric vehicles in microgrids: Overview on hosting capacity and new controls," IEEE Transactions on Industry Applications, vol. 55, no. 6, pp. 7338-7346, Nov. 2019, doi: 10.1109/TIA.2019.2933800.

[10] J. Lee and G. L. Park, "Charger reservation scheme for electric vehicles based on stable marriage problem adaptation," in ACM International Conference Proceeding Series, 2018, pp. 316-319, doi: 10.1145/3301551.3301587.

[11] J. Lee and G. L. Park, "Service time analysis for electric vehicle charging infrastructure," International Journal of Electrical and Computer Engineering (IJECE), vol. 8, no. 2, pp. 818-824, Apr. 2018, doi: 10.11591/ijece.v8i2.pp818-824.

[12] K. Rahbar, C. C. Chai, and R. Zhang, "Energy cooperation optimization in microgrids with renewable energy integration," IEEE Transactions on Smart Grid, vol. 9, no. 2, pp. 1482-1493, Mar. 2018, doi: 10.1109/TSG.2016.2600863.

[13] J. Zhang, J. Jorgenson, T. Markel, and K. Walkowicz, "Value to the grid from managed charging based on California's High renewables study," IEEE Transactions on Power Systems, vol. 34, no. 2, pp. 831-840, Mar. 2019, doi: 10.1109/TPWRS.2018.2872905.

[14] A. A. Daoud, A. F. Abouzeid, and S. S. Dessouky, "Offshore wind power integration to support weak grid voltage for industrial loads using VSC-HVDC transmission system," International Journal of Electrical and Computer Engineering (IJECE), vol. 11, no. 3, pp. 1876-1885, Jun. 2021, doi: 10.11591/ijece.v11i3.pp1876-1885.

[15] J. Lee and G. L. Park, "Renewable energy matching to consumer entities based on a maximum flow model," IOP Conference Series: Materials Science and Engineering, vol. 752, no. 1, p. 12007, Feb. 2020, doi: 10.1088/1757-899X/752/1/012007.

[16] A. Al Hadi, C. A. S. Silva, E. Hossain, and R. Challoo, "Algorithm for demand response to maximize the penetration of renewable energy," IEEE Access, vol. 8, pp. 55279-55288, 2020, doi: 10.1109/ACCESS.2020.2981877.

[17] T. Logenthiran, D. Srinivasan, and T. Z. Shun, "Demand side management in smart grid using heuristic optimization," IEEE Transactions on Smart Grid, vol. 3, no. 3, pp. 1244-1252, Sep. 2012, doi: 10.1109/TSG.2012.2195686.

[18] X. Z. Xia, Z. Mingke, H. Yu, J. M. Guerrero, and J. C. Vasquez, "Coordinated primary and secondary frequency support between microgrid and weak grid," IEEE Transactions on Sustainable Energy, vol. 10, no. 4, pp. 1718-1730, Oct. 2019, doi: 10.1109/TSTE.2018.2869904.

[19] D. A. Aviles, J. Pascual, L. Marroyo, P. Sanchis, and F. Guinjoan, "Fuzzy logic-based energy management system design for residential grid-connected microgrids," IEEE Transactions on Smart Grid, vol. 9, no. 2, pp. 530-543, Mar. 2018, doi: 10.1109/TSG.2016.2555245.

[20] A. T. Eseye, M. Lehtonen, T. Tukia, S. Uimonen, and R. John Millar, "Machine learning based integrated feature selection approach for improved electricity demand forecasting in decentralized energy systems," IEEE Access, vol. 7, pp. 91463-91475, 2019, doi: 10.1109/ACCESS.2019.2924685.

[21] S. E. Razavi, A. Arefi, G. Ledwich, G. Nourbakhsh, D. B. Smith, and M. Minakshi, "From load to net energy forecasting: shortterm residential forecasting for the blend of load and PV behind the meter," IEEE Access, vol. 8, pp. 224343-224353, 2020, doi: 10.1109/ACCESS.2020.3044307.

[22] D. Benda, X. Chu, S. Sun, T. Q. S. Quek, and A. Buckley, "Renewable energy sharing among base stations as a min-cost-maxflow optimization problem," IEEE Transactions on Green Communications and Networking, vol. 3, no. 1, pp. 67-78, Mar. 2019, doi: 10.1109/TGCN.2018.2876005.

[23] C. M. Fernandez, A. A. Freitas, A. N. Morais, T. M. Lima, and P. D. Gaspar, "Fleet management optimization in car rental industry: decision aid models for Logistics improvement," in 2020 International Conference on Decision Aid Sciences and Application, DASA 2020, Nov. 2020, pp. 168-172, doi: 10.1109/DASA51403.2020.9317221. 